\title{
A Survey and Comparison of Various Cloud Simulators Available for Cloud Environment
}

\author{
Ramandeep Kaur ${ }^{1}$, Navtej Singh Ghumman ${ }^{2}$ \\ M.Tech Student, Department of CSE, SBSSTC, Ferozepur, Punjab ${ }^{1}$ \\ Assistant Professor, Department of CSE, SBSSTC, Ferozepur, Punjab ${ }^{2}$
}

\begin{abstract}
Cloud computing is new technology with its ease of access and a variety of applications. If offers various types of services to the user, such as software as a service, platform as a service and hardware as a service or infrastructure as a service. The deployment of real cloud for commercial use is quite expensive. Therefore, various kinds of simulators such as CloudSim, GroudSim, CDOSim, GreenCloud are available in the market for cloud environment. In this paper, we have done a study and analysis of various simulators available for cloud computing.
\end{abstract}

Keywords: Cloud Computing, Services, Features, Advantages, Cloud Simulator, Comparison

\section{INTRODUCTION}

Cloud Computing is an internet technology with its the public cloud. It is also called as on-premise unique characteristics such as self-service, broad network infrastructure.

access, large pool of online resources, rapid elasticity, pay per use model etc. [1] [2]. It is high performance computing, which was earlier considered expensive, but it has become affordable for SMEs (small medium enterprises).

Unlike traditional computing, in which all the documents are created on a single PC and they remain stored there only and can be accessed only there or via a PC connected to it through a network. But, it can't be accessed outside the network. Therefore, it's PC-centric.

In case of cloud computing, we create, edit and store everything on the cloud and can be accessed through any device at any place and you just need internet connection. Therefore, it can be called as document centric.

Cloud computing provides the end-user with software to create applications, platform to deploy them and infrastructure to run and maintain. Cloud computing offers a variety of applications such as business applications (ERP, CRM), technical applications (brain imaging), social applications (Facebook, Twitter, Gmail) etc. Cloud computing is growing day by day and is being used in almost every day to day applications.

\section{Cloud Deployment Models}

There are various types of deployment models available for cloud such as [3][4] as shown in Fig. 1:

\section{A. Public Cloud}

Public cloud is open for public use. Anyone can access the cloud freely or pay per use basis. Amazon EC2 service, Gmail, Google App Engine, Facebook is an example of public clouds. It is also known off-premise infrastructure.

\section{B. Private Cloud}

\section{Community Cloud}

It is owned by a group of organizations or a community having similar business needs. This type of cloud is accessible only by the members of the organizations who have ownership. Thus, any outsider is not allowed the access and thus its more secure than public cloud but less secure than private cloud.

\section{Hybrid Cloud}

It can be thought of as any combination of public, private and community cloud [5]. Since, it has the features of public, private and hybrid cloud, hence the word, 'hybrid'.

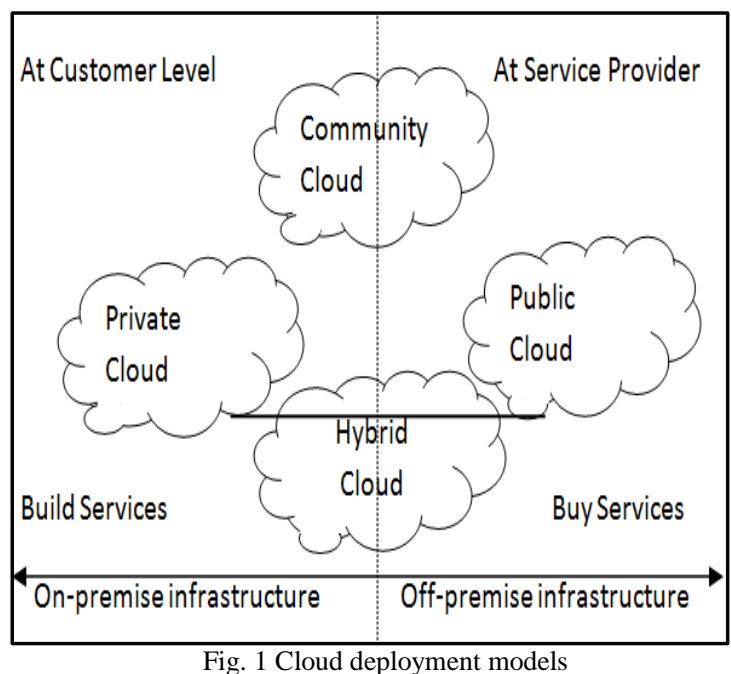

\section{III.SIMULATION OF ClOUd ENVIRONMENT}

A private cloud is owned by some organization and its members are only allowed to access it. ERP cloud is purchased by an organization and tailored according to the needs of an organization. Therefore, it is more secure than

\section{A. Why Simulation is required?}

The real deployment of cloud is very expensive if we need it for personal use because efficient resource utilization is not possible. Research scholars need to test 
their algorithms in a cloud environment. Various cloud simulators have come up to handle the issue. Simulators environment. It is becoming popular day by day due to its offer a dependent and controlled cloud environment for extendibility.

conducting experiments.

\section{B. Pros of Simulation}

The pros of Simulation are as follows:

1) Reduces the risk involved: Since we are using the simulators and not the real cloud, so users can test and verify the results and identify the risk involved in the implementation of design or any parameter. Here no cost of running the experiment is there since it's just a simulation.

2) Do not require much of the expertise: Use of simulation tool does not any kind of expertise related to deployment of cloud. The user just needs to have programming skills for
manipulating the code according to his needs and rest all is left to the simulator.

3) No capital cost involved: In case of cloud built on CloudSim and with GUI interface as shown in Fig. computing, there is a shift from capital cost to 3 . operational cost. If we use cloud simulator, there is no capital cost involved.

4) Provides better results: We can run the simulation ' $\mathrm{n}$ ' no of times by changing the input values ' $n$ ' times and thus produce output too precise.

Thus, above mentioned pros clearly show that use of simulators is a better option than deploying a real cloud for research scholars.

\section{Cloud Simulation Tools}

\section{A. CloudSim}

CloudSim [7] is a famous simulator for cloud parameters developed in the CLOUDS Laboratory, at the Computer Science and Software Engineering Department of the University of Melbourne. CloudSim architecture is shown in Fig. 2.

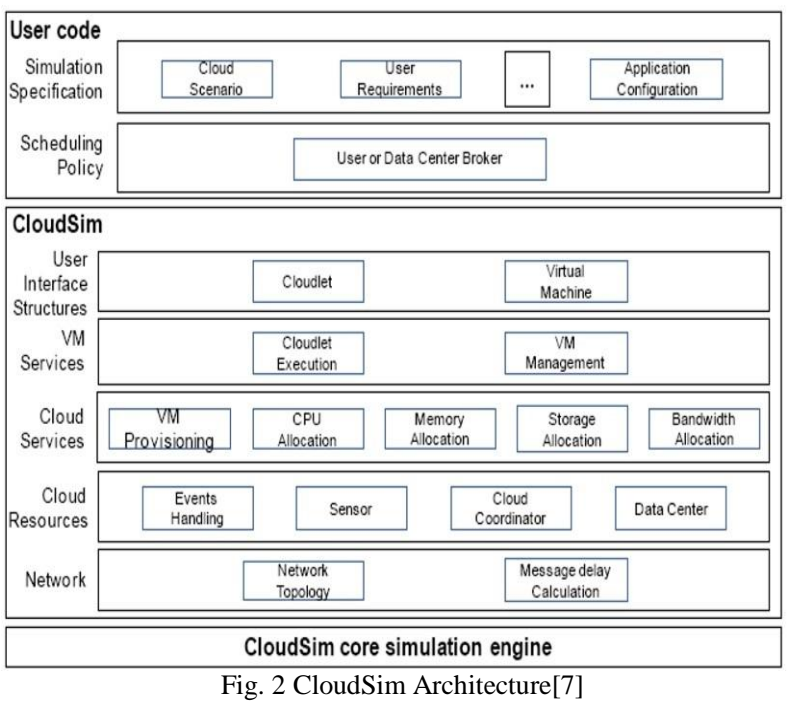


adding any other communication protocol. This simulator is commercial i.e. user need to buy for its full functionality and this is the main limitation as opposed to other simulators.

\section{E. SmartSim}

It is the only simulator [11] built for mobile cloud computing till date. It is used for modelling and simulating mobile cloud computing applications in mobile. It uses resource provision, evaluation method for resource utilization in SMD (smart mobile device).

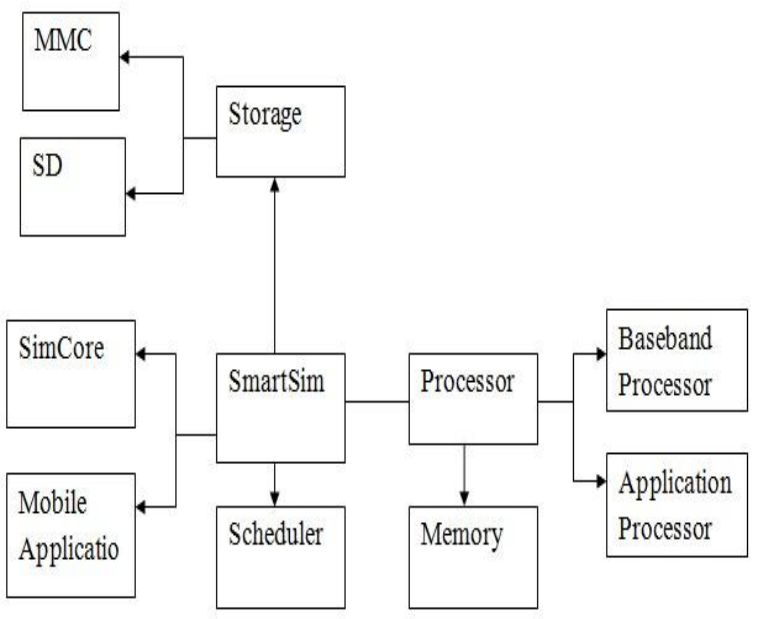

Fig. 4 Block diagram of SmartSim

\section{F. GroudSim}

It is a discrete event simulation tool for cloud and grid computing. It [10] is made specifically for scientific applications in cloud and grid computing. It has Java as an underlying language. It has one unique feature called as GroundEntity which has its own error definitions which the user can change at the time of error occurrence.

\section{G. DCSim}

It is a data center simulation tool for dynamic resource provisioning. It consists of multiple interconnected hosts and each host consist of scheduler and resource management policy. It simulates data center with a central management system.

\section{COMPARISON OF VARIOUS SIMULATORS}

In the previous section, we have discussed about cloud simulators. In this section, we are going to compare them on the basis of various parameters such as [10] underlying programming language, energy model,federation policy, cost etc. as presented in Table 1 .

Various parameters discussed in above table as explained as follows:

1) Underlying platform: It refers to the existing framework as some cloud simulators are built upon some existing simulation framework, i.e. they are extended form of existing one. For example, CloudSim is built upon SimJava.

2) Availability: It means that whether the simulator is open source or commercially available. CloudSim is open source and MDCSim is commercial.

3) Programming language: It refers to the programming language skills needed by user to learn in order to work with the particular simulator. In case of CloudSim, user only needs to have Java programming skills.

4) Cost: User wants to work on cost modelling as cloud is pay per use model so he must make the right choice of the simulator.

5) GUI (Graphical User Interface): It refers to the type of interface that the simulator offers to the user. CloudAnalyst has a GUI interface which is quite attractive and easy to use. Moreover, CloudAnalyst produces results in graphical form.

6) Communication model: It decides how the communication takes place in the simulator. In case of CloudSim, communication between and within the data centers takes place by message passing.

7) Simulator Time: It is the time taken by the simulator to execute simulation. Some simulators have simulation time in seconds such as CloudSim and in minutes such as DCSim.

8) Energy model: It is used to calculate energy consumption by huge data centers. GreenCloud is specially built for this purpose.

9) Federation policy: Since cloud is a vast heterogeneous, distributed network, so they are different cloud providers all over the globe. In order to allow coordination among them, the federation policy supports networking between them.

Table 1. Comparison of various cloud simulators

\begin{tabular}{|l|l|l|l|l|l|l|l|l|l|}
\hline Simulator & $\begin{array}{l}\text { Underlying } \\
\text { Platform }\end{array}$ & Available & $\begin{array}{l}\text { Programming } \\
\text { Language }\end{array}$ & Cost & GUI & $\begin{array}{l}\text { Communication } \\
\text { Model }\end{array}$ & $\begin{array}{l}\text { Simulation } \\
\text { Time }\end{array}$ & $\begin{array}{l}\text { Energy } \\
\text { Model }\end{array}$ & $\begin{array}{l}\text { Federation } \\
\text { policy }\end{array}$ \\
\hline CloudSim & SimJava & $\begin{array}{l}\text { Open } \\
\text { Source }\end{array}$ & Java & Yes & No & Limited & Second & Yes & Yes \\
\hline CloudAnalyst & CloudSim & $\begin{array}{l}\text { Open } \\
\text { Source }\end{array}$ & Java & Yes & Yes & Limited & Second & Yes & Yes \\
\hline GreenCloud & NS-2 & $\begin{array}{l}\text { Open } \\
\text { Source }\end{array}$ & C++, octal & No & Limited & Full & Minute & Yes & No \\
\hline MDCSim & CSIM & Commercial & Java/C++ & No & No & Limited & Second & Rough & No \\
\hline DCSim & - & $\begin{array}{l}\text { Open } \\
\text { Source }\end{array}$ & Java & Yes & No & No & Minute & No & No \\
\hline GroudSim & - & $\begin{array}{l}\text { Open } \\
\text { Source }\end{array}$ & Java & No & No & Limited & Second & No & No \\
\hline SmartSim & CloudSim & $\begin{array}{l}\text { Open } \\
\text { Source }\end{array}$ & Java & Yes & No & Limited & Second & Yes & Yes \\
\hline
\end{tabular}




\section{VI.CONCLUSION}

Cloud computing is growing at a much faster rate. Also, it's faced with so many challenges in its infancy. Order to carry out a fundamental research in cloud computing, cloud simulators are considered to be a better option rather than real deployment of cloud. In this paper, we presented a survey of various cloud simulators and also done a comparison on the basis of some parameters.

\section{ACKNOWLEDGMENT}

The Success of this work would have been uncertain without the help and guidance of a dedicated group of people in our institute SBS State Technical Campus, Ferozepur. I would like to express the deepest appreciation to my guide. I am also thankful to my college friends for their support.

\section{REFERENCES}

[1] http://www.explainthatstuff.com/cloud-computing introduction.html. [Accessed (May 3, 2015)], "Woodford, Chris. (2009) Cloud computing."

[2] Mell, Peter and Grance, Tim, "The NIST definition of cloud computing", National Institute of Standards and Technology, 2009,vol 53, p.50, Mell2009.

[3] http://www.davidakka.com/uncategorized/cloud-modelswhat $\%$ E2\%80\%99s-right-for-you/, "Cloud models-what"s right for you".

[4] Marinos, Alexandros, and Gerard Briscoe, "Community cloud computing. ”Cloud Computing. Springer Berlin Heidelberg, 2009, pp.472-484.

[5] http://searchcloudcomputing.techtarget.com/definition/hybridcloud, "hybrid cloud".

[6] Kumar, Parveen, and Anjandeep Kaur Rai. "An Overview and Survey of Various Cloud Simulation Tools." Journal of Global Research in Computer Science, Vol 5(1) , pp: 24-26, 2014.

[7] Calheiros, Rodrigo N., Rajiv Ranjan, Anton Beloglazov, Cesar AF De Rose, and Rajkumar Buyya. "CloudSim: a toolkit for modeling and simulation of cloud computing environments and evaluation of resource provisioning algorithms. "Software: Practice and Experience 41, pp: 23-50, 2011.

[8] B.Wickremasinghe, "CloudAnalyst: A CloudSim based Tool for Modeling and Analysis of Large Scale Cloud Computing Environments", MEDC Project Report, 2009.

[9] S. Lim, B. Sharma, G .Nam, E. K. Kim, and C. R. Das "MDCSim: A Multi-tier Data Center Simulation Platform" Cluster Computing and Workshops, 2009.

[10] M. Shiraz, A. Gani, R. H. Khokhar, E Ahmed, "An Extendable Simulation Framework for Modeling Application Processing Potentials of Smart Mobile Devices for Mobile Cloud Computing”, 10th International Conference on Frontiers of Information Technology, 2012

[11] S. Ostermann, K. Plankensteiner, R. Prodan, "Th. Fahringer, "GroudSim: An Event Based Simulation Framework for Computational Grids and Clouds", Euro-Par 2010 Parallel Processing Workshops Lecture Notes in Computer Science Volume 6586, pp: 305-313, 2011.

[12] Ahmed, Arif, and Abadhan Saumya Sabyasachi. "Cloud computing simulators: A detailed survey and future direction." In Advance Computing Conference (IACC), 2014 IEEE International, pp. 866872. IEEE, 2014 